\title{
Three-Dimensional Stereophotogrammetric Analysis of Adults Affected by Dravet Syndrome
}

\author{
Valentina PUCCIARELLI ${ }^{1}$, Elena PIAZZA ${ }^{2,3}$, Francesca RAGONA ${ }^{2}$, \\ Daniele GIBELLI ${ }^{1}$, Tiziana GRANATA ${ }^{2}$, Claudia DOLCI ${ }^{1}$, Chiarella SFORZA ${ }^{1}$ \\ 1 Dipartimento di Scienze Biomediche per la Salute, Università degli Studi di Milano, Milan, Italy; \\ 2 Dipartimento di Neuroscienze Pediatriche, Istituto Neurologico C. Besta, Milan, Italy; \\ ${ }^{3}$ Dipartimento di Neuropsichiatria Infantile, Istituto Neurologico C. Mondino, Pavia, Italy
}

DOI: $10.15221 / 17.163$ http://dx.doi.org/10.15221/17.163

\begin{abstract}
Dravet syndrome (DS) is a rare and severe form of epilepsy, associated with mutations of SCN1A gene, encoding for the sodium channel voltage-dependent Nav.1.1. Epilepsy appears in the first year of life in an otherwise healthy infant; seizures may be focal, unilateral or generalized and facilitated by fever. The disease is progressive and during its course neurological signs change, comorbidities appear and cognitive deficits become highly disabling. Currently, early diagnosis of the syndrome is possible, nevertheless, up today, many adult cases remain not yet diagnosed, especially if the clinical childhood history is fragmented. A preliminary stereophotogrammetric morphometric evaluation of facial soft tissues of six Dravet syndrome patients ( 3 males, 3 females, aged between 16 and 37 years) was performed with the aim of facilitating the recognition of the syndrome also in adult patients and identifying morphological common facial features in affected patients. The three-dimensional coordinates of a set of facial landmarks, identified on the faces of the patients, were collected and compared to the coordinates of a group of reference subjects, paired for age, sex and ethnicity and previously acquired. From these coordinates, linear measurements were performed and z-scores were computed. The analysis of $z$-scores showed that patients had a reduction of mandibular ramus length $(z$-score $=-1.7)$ and a consequent reduction of the ratio between the anterior and posterior facial heights $(z$-score $=-1.3)$. Furthermore, 4 of the 6 patients had a reduction of the labial philtrum width. Despite the reduced number of patients, this preliminary report shows the presence of dysmorphic features among them. These results could give a support for a correct diagnosis in adult patients and could indicate a possible role of SCN1A or close genes in facial morphogenesis.
\end{abstract}

Keywords: Dravet syndrome, stereophotogrammetry, facial anatomy

\section{Introduction}

Dravet syndrome is a rare form of epilepsy, and is accompanied by impaired psychomotor and neurologic development, occurring in the first year of life in otherwise healthy children. Initially described in 1978 [1] as "severe myoclonic epilepsy of infancy" (SMEI); in the following years several authors reported similar cases in Europe and in Japan [2,3]. In 1989 the eponym "Dravet syndrome" was proposed, because it became obvious that the epilepsy was not limited to infancy and childhood but it persisted throughout adulthood [4,5]. (OMIM \#607208, Commission on Classification and Terminology of the International League Against Epilepsy, 1989). The disease onset is during the first year of life and is marked by repeated generalized or unilateral clonic (hemiclonic with alternating side) seizures, usually triggered by fever. Seizures are often prolonged, recur in clusters and may evolve into status epilepticus [6]. A stagnation in development becomes evident from the second year of life. Language starts developing at a normal age, but it progresses very slowly and many patients do not reach the stage of constructing elementary sentences. Patients' fine motor abilities do not develop well. They are disturbed by segmental myoclonus and by a poor eye-hand coordination. Learning disabilities also depend on lack of attention, hyperactivity and oppositive behavior; all of which are often present in these patients. In a minority of cases autism spectrum traits have also been reported [7-9].

During the disease course neurologic signs appear in most of the patients, as well as hypotonia, ataxia $(60 \%)$, pyramidal signs $(20 \%)$, uncoordinated movements and interictal myoclonus [6]. In the last stage, after the age of 6-10 years, there is a clinical stabilization of the disease. 
In adulthood seizures persist but are less frequent: convulsive seizures are rarely prolonged and usually confined to sleep; absence and myoclonic seizures can usually disappear, focal seizures persist or decrease.

The clinical picture in adulthood is dominated by cognitive disability, in the most of the cases associated with behavioral disorders, as well as agitation, irritability and obsessive traits. In some of the patients autistic features are described. Adult patients present extrapyramidal signs (hypomimia, bradykinesia, abnormal latency in initiating movements) and myoclonus. Some patients present different degree of gait impairment, determined by the combination of extrapiramidal signs and orthopedic anomalies (kyphosis, scoliosis, flat feet and valgus knees) [10-12].

In 2001, for the first time, the presence of heterozygous mutations in the SCN1A gene, coding for the a-subunit of the neuronal voltage-gated sodium ion channel, type1 ( NaV 1.1) was identified in $7 \mathrm{DS}$ patients [13]. After the first description, several studies confirmed the presence of mutations in the SCN1A gene in DS patients. Currently, the frequency of mutations is detected around $70-80 \%$; truncating mutations account for nearly $50 \%$ of the abnormalities, while the remaining ones include splice site and missense mutations. Intragenic deletions and whole gene deletions, including SCN1A and contiguous genes, account for $2-3 \%$ of all cases. About $12.5 \%$ of all cases exhibit no point mutations [14-16].

In about $95 \%$ of patients, mutations are de novo, but familial SCN1A mutations may also occur $[17,18]$. Somatic mosaic mutations have been reported in some patients and should be taken into account when estimating the recurrence [19,20]; furthermore mosaic SCN1A mutations might contribute to explain the phenotypic variability seen within the same family.

The aim of this preliminary study is to facilitate the recognition of the syndrome also in adult patients, identifying morphological common facial features among them.

Different human syndromes such as Down's and Marfan and also epileptic ones, like Glut-1 deficiency, are characterised by typical facial features, whose recognition can improve the detection of the disease, both in young and adult subjects [21-26]. Currently, facial features can be easily investigated through an anthropometric approach by means of user-friendly and not invasive optical instruments such as stereophotogrammetry and laser scanners $[27,28]$.

At this purpose, the three-dimensional facial morphology of a group of DS patients was analysed. The identified morphological features may be useful for the recognition of the disease in adults not previously diagnosed and in cases of unusual or variable presentation of the disease.

\section{Method}

\subsection{Involved patients and control subjects}

Six patients (three females and three males), aged from 16 to 37 years and affected by DS, were analyzed. All of them had received a clinical diagnosis of DS during childhood, according to the criteria proposed by the International League Against Epilepsy [5]. The clinical diagnosis had also been confirmed by a mutational analysis of the SCN1A gene executed with Sanger method, denaturing high performance liquid chromatography (DHPLC). Four of them presented a missense mutation, two of them a nonsense mutation. The study also included 396 control subjects, of the same ethnicity, sex and age of patients. Details regarding the analyzed patients and reference subjects are shown in Table 1. All control subjects did not have facial deformities and/or a previous history of facial surgery or traumas.

All the procedures were performed according to the tenets of the Declaration of Helsinki and had also been approved by the local ethic committee. They were safe and did not imply any kind of risk. Before performing every acquisition the procedures were explained and a written informed consent was collected from the interested subjects or the parents/ legal tutors.

Table 1. Patients analysed in this study and number of corresponding control subjects used for comparisons.

\begin{tabular}{|c|c|c|c|}
\hline & gender & age (years) & \# of reference subjects \\
\hline patient \#1 & female & 16 & 30 \\
\hline patient \#2 & female & 17 & 13 \\
\hline patient \#3 & female & 26 & 23 \\
\hline patient \#4 & male & 18 & 178 \\
\hline patient \#5 & male & 32 & 76 \\
\hline patient \#6 & male & 37 & 76 \\
\hline
\end{tabular}




\subsection{Data collection and analysis}

Data collection was performed through a stereophotogrammetric system, VECTRA M3 (Canfield Scientific Inc, Fairfield, USA). The instrument is composed of three couples of coordinate cameras that simultaneously acquire facial images from different points of view and allow to obtain, in a $3.5 \mathrm{~ms}$ capture time, 3D facial reconstructions with a $1.2 \mathrm{~mm}$ geometry resolution [29].

The acquisition phase was preceded by the identification and marking of a set of 50 standardized landmarks on the patients and controls faces. Landmarks were gently drawn using a black liquid and biocompatible eyeliner, while the subjects were seated in front of the stereophotogrammetric system [27]. Once the facial reconstructions were obtained, all the landmarks were digitized on them and a subset of 13 , located both in the midline and in the corresponding hemifaces, was chosen to extract the corresponding 3D coordinates. These coordinates were used to calculate a series of linear measurements.

Details about the set of landmarks used in this study are listed below; a similar subset was used in a previous study about Glut-1 deficiency syndrome [25]. Figure 1 illustrates their position on the face.

- Trichion (Tr): in the midline, at the attachment of the hair;

- Nasion (N): in the midline, at the suture between frontal and nasal bones;

- Pronasale (Prn): in the midline; at the tip of the nose;

- Subnasale (Sn): in the midline; at the end of columella;

- Christa philtri (Cph): paired; at the upper point of the labial philtrum;

- Pogonion (Pg): in the midline; the most prominent point of the chin;

- Exocanthion (Ex): paired; the outer eye commissure;

- Zygion (Zy): paired; the most prominent point of the zygomatic arch;

- Tragion (T): paired; in the middle of tragus;

- Gonion (Go): paired; at the angle of the mandible.

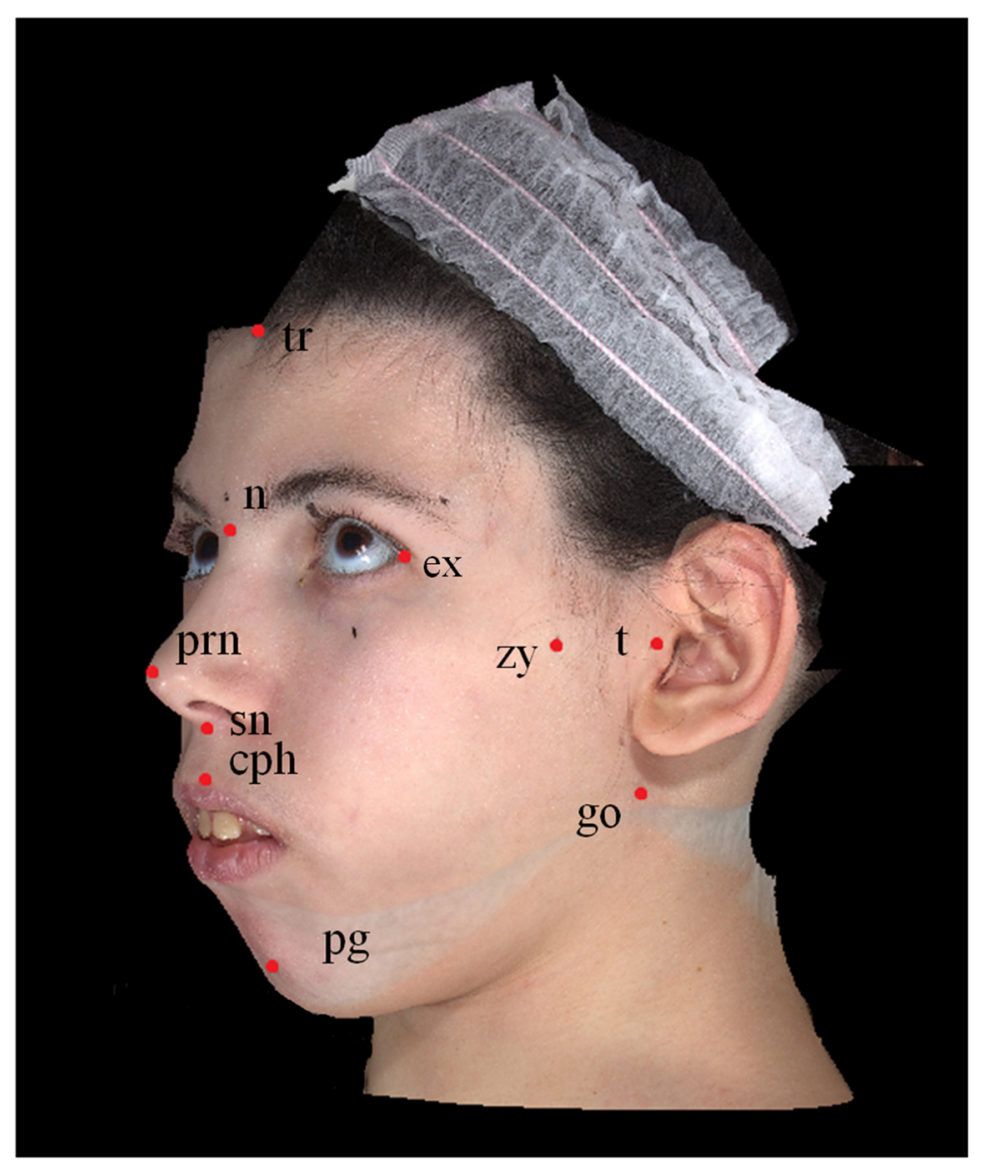

Fig. 1. Landmarks digitized on a patient's face. 
For each subject a set of 15 linear distances and two distance ratios were calculated. Linear distances were expressed in millimetres, ratios in percentage.

Performed measurements are listed below; I and $r$ indicate left and right facial sides respectively.

Horizontal distances include:

- Biocular width (Exr-ExI);

- Skull base width $\left(T_{r}-T_{1}\right)$;

- Mandibular width (Gor-Goı);

- Facial width $\left(Z y_{r}-Z y_{1}\right)$;

- Mouth width $\left(\mathrm{Ch}_{\mathrm{r}}-\mathrm{Ch}_{1}\right)$;

- Philtrum width ( $\mathrm{Cph}_{\mathrm{r}}-\mathrm{Cph}$ ).

Vertical distances include:

- Length of the upper third of the face (Tr-N);

- Length of the middle third of the face (N-Sn);

- Length of the lower third of the face $(\mathrm{Sn}-\mathrm{Pg})$;

- Morphological height of the face (N-Pg);

- Mandibular ramus length (T-Go).

Sagittal distances include:

- Mandibular body length (Pg-Go);

- Upper facial third depth (N-T);

- Depth in the maxillary region (Sn-T);

Ratios:

- Mandibular sagittal plane depth (Pg-T).

- Posterior to anterior facial heights ratio (T-Go/Sn-Pg);

- Facial width/height (T-T/N-Pg).

A custom software was used to automatically perform all the calculations starting from the 3D coordinates. Once obtained the linear distances, $z$ score values were computed. The calculation was performed subtracting from the single measurement of each patient the corresponding mean measurement of the control group and dividing by the relevant standard deviation.

For patients younger than 18 years of age, $z$ score calculation was performed using control subjects exactly paired for age; older patients were matched with controls with an age span of few years, compared to the patient's age. The age intervals for controls older than 18 years were 18-25; 26-30 and 31-40 years. Subsequently, the mean $z$ scores and corresponding standard deviations were calculated for all measurements considered.

\section{Results}

Tables $2 a$ and $2 b$ show the descriptive statistics of the $z$ scores calculated for this study. In particular, Table $2 \mathrm{a}$ shows the results for the horizontal and vertical distances, while Table $2 \mathrm{~b}$ shows the results for the sagittal distances and the ratios.

Table 2a. Descriptive statistics of the z scores calculated for horizontal and vertical distances. Measurements differing on average 1.5 SD or more from the reference values are marked with *.

\begin{tabular}{|c|c|c|c|c|c|c|c|c|c|c|c|}
\hline & Exr-Ex। & $T_{r}-T_{1}$ & Gor-Gol & $Z y_{r}-Z y_{1}$ & $\mathrm{Ch}_{\mathrm{r}-\mathrm{Ch}}$ & Tr-N & $\mathrm{Cph}_{\mathrm{r}}-\mathrm{Cph}$ & $\mathrm{N}-\mathrm{Sn}$ & $\mathrm{Sn}-\mathrm{Pg}$ & $\mathrm{N}-\mathrm{Pg}$ & T-Go \\
\hline $\mathrm{F} 1$ & -0.2 & 0.3 & 0.3 & -0.1 & -0.1 & -0.6 & 2.1 & 0.9 & -0.7 & 0.3 & -2.9 \\
\hline $\mathrm{F} 2$ & -0.1 & -0.1 & 0.6 & 0 & 0.3 & -1 & -0.9 & -0.8 & 0.8 & 0 & -1.6 \\
\hline F3 & 0.2 & 0.2 & 0.3 & 0.1 & -0.7 & 1.8 & -1.9 & 0.1 & 1.4 & 0.5 & -1.4 \\
\hline M1 & -0.8 & -0.8 & -0.3 & -3.2 & -0.9 & -0.3 & -2 & 0.2 & -0.6 & 0 & -0.9 \\
\hline M2 & -3 & -1.2 & 1.9 & -2.3 & -1.2 & -0.2 & 0.1 & 1.2 & 0.1 & 1.2 & -2 \\
\hline M3 & -0.8 & -3 & -0.6 & -1.6 & 0.7 & -1.5 & -0.6 & -1.1 & 1.5 & 0.4 & -1.7 \\
\hline Mean & -0.8 & -0.8 & 0.4 & -1.2 & -0.3 & -0.3 & -0.5 & 0.1 & 0.4 & 0.4 & $-1.7^{*}$ \\
\hline$S D$ & 1.1 & 1.3 & 0.9 & 1.4 & 0.8 & 1.1 & 1.5 & 0.9 & 1 & 0.4 & 0.7 \\
\hline Min & -3 & -3 &.,- 6 & -3.2 & -1.2 & -1.6 & -2 & -1.1 & -0.7 & 0 & -2.9 \\
\hline Max & 0.2 & 0.3 & 1.9 & 0.1 & 0.7 & 1.8 & 2.1 & 1.2 & 1.5 & 1.2 & -0.9 \\
\hline
\end{tabular}


Table 2b: Descriptive statistics of the $z$ scores calculated for sagittal distances and ratios.

\begin{tabular}{|l|c|c|c|c|c|c|}
\hline & Pg-Go & N-T & Sn-T & Pg-T & T-Go/Sn-Pg & T-T/N-Pg \\
\hline F1 & 2 & 0 & 0.1 & 0.2 & -1.3 & -0.4 \\
\hline F2 & 0.9 & -0.6 & -0.8 & -1.1 & -1.6 & -0.1 \\
\hline F3 & 1.8 & 0.3 & 0.3 & 0.3 & -1.8 & -1.1 \\
\hline M1 & 0 & -1 & -2.6 & -2.2 & -0.7 & -0.5 \\
\hline M2 & 0.9 & -0.6 & -1.7 & -1.3 & -1 & -1.6 \\
\hline M3 & 0.7 & -1.6 & -2 & -2.6 & -1.3 & 0 \\
\hline Mean & 1.1 & -0.6 & -1.1 & -1.1 & -1.3 & -0.6 \\
\hline SD & 0.7 & 0.7 & 1.2 & 1.2 & 0.4 & 0.6 \\
\hline Min & 0 & -1.6 & -2.6 & -2.6 & -1.8 & -1.6 \\
\hline Max & 2 & 0.3 & 0.3 & 0.3 & -0.7 & 0 \\
\hline
\end{tabular}

On average the comparison between DS patients and reference subjects did not show important differences for what concerns the horizontal and sagittal distances, since only mean patient $z$ scores at least 1.5 SD different from the control values are considered remarkable (by definition the average $\mathrm{z}$ score of controls is $=0$ and their $\mathrm{SD}$ is $=1$ ).

On the other hand, among the vertical distances, the mandibular ramus length T-Go resulted reduced in the patients. Subsequently, the ratio between their posterior and anterior facial height resulted reduced too.

Furthermore, despite the absence of a mean z-score outside the $\pm 1.5 \mathrm{SD}$ interval, four patients presented a reduction of the labial philtrum width ( $z$ scores respectively $-0.9,-1.9,-2,-0.6$ ).

Even if the reduced dimension of the sample prevented from statistical considerations, a different trend between males and females for facial width and depth in the maxillary region was evident: female faces seemed to be in the range of the controls, while male ones differed more from the reference values.

Figure 2 shows an example of geometric representation of the average profile of the patients, compared to a geometric representation of the average profile of the controls. It is possible to appreciate, in patients, the reduction of the mandibular ramus length and the corresponding reduced ratio between posterior and anterior facial heights.
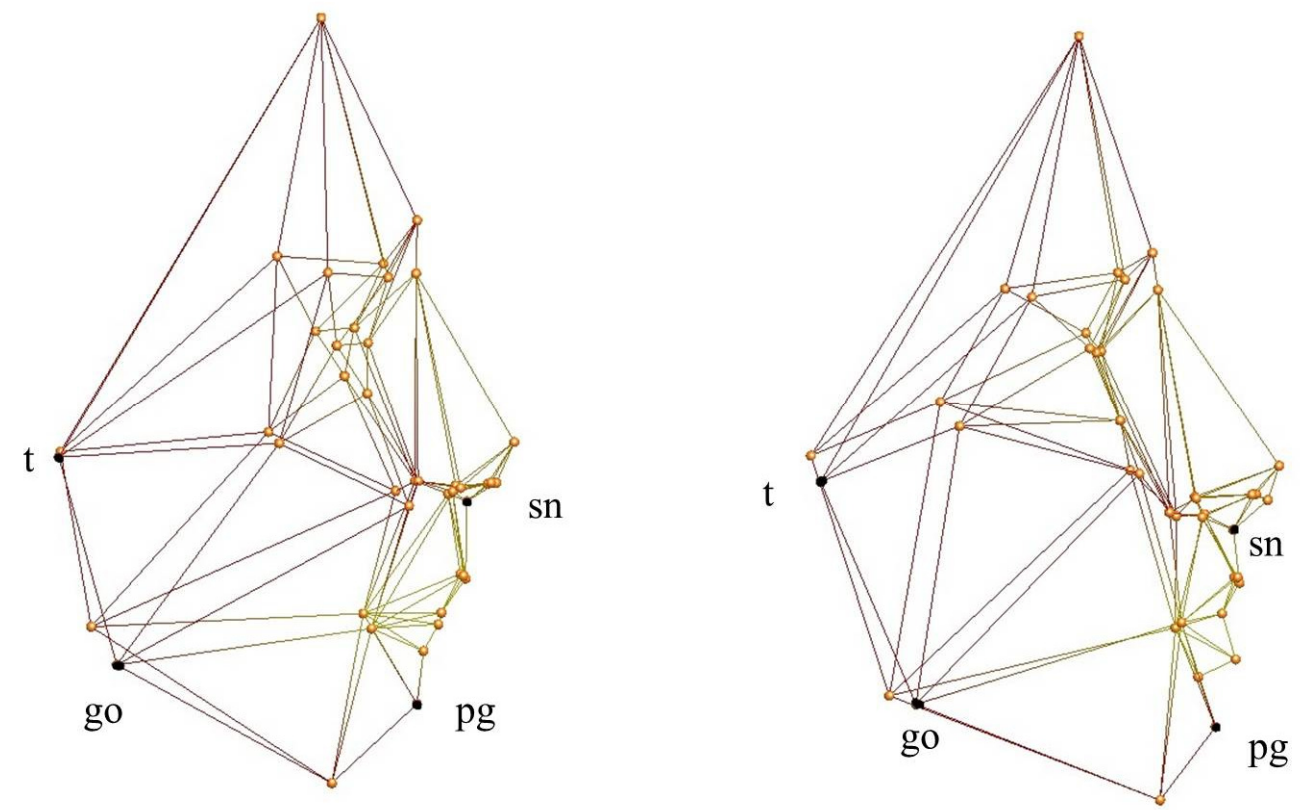

Fig. 2. a) Geometric representation of the average profile of the patients;

b) Geometric representation of the average profile of the controls,

t-go: mandibular ramus length (posterior facial height); sn-pg: anterior facial height. 


\section{Discussion}

DS can be considered a multisystem chronic disorder, with a childhood onset, that continues during adulthood with a different clinical picture. Up today some adult cases can remain undiagnosed, for the absence of a documented childhood clinical history, especially for those patients entrusted in institutional care and for the reduced availability of longitudinal data about adult phenotypes $[20,30]$. Nevertheless the recognition of this syndrome in adult patients has a great importance, not only from a clinical point of view, but also for the familial management which must be oriented to the application of seizures reduction strategies and to a global approach to the patient needs and quality of life [31]. In order to recognize facial traits common among the patients, which could represent "soft markers" of the disease and could be helpful for the diagnosis in unrecognised adult patients, in this preliminary study a 3D stereophotogrammetric evaluation of the facial soft tissues of six DS patients was performed.

An anthropometric approach for the assessment of the facial morphology of syndromic patients is, indeed, advisable: many syndromes are characterised by a more or less evident facial morphology that can guide the clinicians towards the diagnosis, both in children and in adults $[23,24,26]$. Moreover in presence of a certain pathology, facial morphology can be affected in a not apparently appreciable way and becomes manifest thanks to a 3D facial anthropometric assessment [28].

Among the different anthropometric instruments currently available, optical instruments, such as stereophotogrammetry, offer a good opportunity to evaluate the 3D facial aspect, in a well defined, accurate and reproducible way [32]. Furthermore stereophotogrammetry is safe, fast and not invasive and permits to acquire also scarcely or not collaborative patients [27].

A previous attempt to define the facial morphology of child DS patients using photogrammetry was performed in 2011 by Nolan et al. [33]. In this study a series of ratios between linear measurements was performed by two different operators on the photographic records of a group of 12 DS patients aged 4-18 years. Patients data were compared to the measurements performed on the respective siblings, used as controls. No statistical significant differences were found between patients and their relatives [33]. Our results are in contrast with those of Nolan et al., as we found that, on average, patients have a reduced mandibular ramus length and a subsequent reduction between posterior and anterior facial height, in comparison with control subjects.

This disagreement can be explained by the different methods and measurements (frontal plane 2D photographs by Nolan et al., 3D photographs in the current study), age of the patients (children vs. adults), and reference groups. In particular, in the current preliminary evaluation, each patients was compared to a group of matched controls, while Nolan et al. used only patients' siblings as reference subjects [33]. Additionally, the previous investigation did not assess facial posterior vertical distances [33].

Furthermore, in four out of the six DS evaluated patients, we found a reduction of the labial philtrum width. Alterations of this feature are often associated with syndromic condition [28, 34].

In conclusion, despite the reduced patients number, this preliminary report allowed the identification of dysmorphic facial traits in the faces of adults affected by DS.

Although the present results are encouraging to help the recognition of the syndrome in undiagnosed adults, further studies on a larger patients group are mandatory, in order to provide statistically stronger results and clarify a possible role of SCN1A or close genes clusters in facial morphogenesis.

\section{References}

[1] C. Dravet, "Les épilepsies graves de l'enfant", Vie Méd, Vol. 8, 1978, pp. 543-8.

[2] B. Dalla Bernardina et al., "Epilepsie myoclonique grave de la première annèe". Rev EEG Neurophysiol, Vol. 12, No. 1,1982, pp. 21-5, http://dx.doi.org/10.1016/S0370-4475(82)80004-X.

[3] T. Ogino et al., "Severe myoclonic epilepsy in infancy- Clinico-electroencephalographic and long-term followup studies", Brain Dev, Vol. 8, No.2, 1986, pp.109-10.

[4] https://www.omim.org/entry/607208, accessed on August $1^{\text {st }}, 2017$.

[5] Proposal for revised classification of epilepsies and epileptic syndromes. Commission on Classification and Terminology of the International League Against Epilepsy. Epilepsia, Vol. 30, No.4, 1989, pp. 389-99.

[6] C. Dravet et al. "Severe myoclonic epilepsy in infancy (Dravet syndrome) 30 years later", Epilepsia, Vol. 52, Suppl 2, 2011, pp.1-2, http://dx.doi.org/10.1111/j.1528-1167.2011.02993.x.

[7] R. Caraballo, N. Fejerman "Dravet syndrome: a study of 53 patients", Epilepsy Res, Vol. 70, Suppl. 1, 2006, pp.S231-8, http://dx.doi.org/10.1016/j.eplepsyres.2005.11.026.

[8] M. Wolf et al. "Severe myoclonic epilepsy of infants (Dravet syndrome): natural history and neuropsychological findings", Vol. 47, Suppl. 2, 2006, pp. 45-8, http://dx.doi.org/10.1111/j.15281167.2006.00688.x. 
[9] F. Ragona et al. "Dravet syndrome: early clinical manifestations and cognitive outcome in 37 Italian patients", Brain Dev, Vol. 32, No. 1, 2010, pp. 71-3, http://dx.doi.org/10.1016/j.braindev.2009.09.014.

[10] C. Dravet et al. "Severe myoclonic epilepsy of infancy (Dravet syndrome). In: Nikanorova M., Genton P., Sabers A. (eds). Long-term evolution of epileptic encephalopathies. Paris: John Libbey Eurotext, pp. 29-38.

[11] P. Martin et al. "Severe Myoclonic Epilepsy in Infancy - Adult Phenotype with Bradykinesia, Hypomimia, and Perseverative Behavior: Report of Five Cases", Mol. Syndromol, Vol. 1, No. 5, 2010, pp. 231-8, http://dx.doi.org/10.1159/000326746.

[12] CB. Catarino et al. "Dravet syndrome as epileptic encephalopathy: evidence from long-term course and neuropathology", Brain, Vol. 134, No. 10, 2011, pp. 2982-3010, http://dx.doi.org/ 10.1093/brain/awr129.

[13] L. Claes et al. "De Novo Mutations in the Sodium-Channel Gene SCN1A Cause Severe Myoclonic Epilepsy of Infancy", Am J Hum Gen, Vol. 68, No. 6, 2001, pp. 1327-32, http://dx.doi.org/10.1086/320609.

[14] R. Guerrini. "Dravet syndrome: the main issues", Eur J Paediatr Neurol, Vol. 16, Suppl. 1, 2012, pp. S1-4, http://dx.doi.org/10.1016/j.ejpn.2012.04.006.

[15] J. Hattori et al. "A screening test for the prediction of Dravet syndrome before one year of age", Epilepsia, Vol. 49, No. 4, 2008, pp. 626-33, http://dx.doi.org/10.1111/j.1528-1167.2007.01475.x.

[16] C. Marini, et al. "The genetics of Dravet syndrome", Epilepsia, Vol. 52, No. 2, 2011, pp. 24-9, http://dx.doi.org/10.1111/j.1528-1167.2011.02997.x.

[17] T. Sugawara et al. "Frequent mutations of SCN1A in severe myoclonic epilepsy in infancy", Neurol, Vol. 58, No. 7, 2002, pp. 1122-4.

[18] MH. Meisler et al. "Sodium channel gene family: epilepsy mutations, gene interactions and modifier effects", J Physiol., Vol. 588, Pt. 11, 2010, pp. 1841-8, http://dx.doi.org/10.1113/jphysiol.2010.188482.

[19] C. Depienne et al. "Parental Mosaicism Can Cause Recurrent Transmission of SCN1A Mutations Associated With Severe Myoclonic Epilepsy of Infancy", Human Mutation, Vol. 27, No. 4, 2006, pp. 389, http://dx.doi.org/10.1002/humu.9419.

[20] C. Marini et al. "Mosaic SCN1A Mutation in Familial Severe Myoclonic Epilepsy of Infancy" Epilepsia, Vol. 47, No. 10, 2006, pp. 1737-1740, http://dx.doi.org/10.1111/j.1528-1167.2006.00675.x.

[21] C. Sforza et al. "Abnormal Variations in the Facial Soft Tissues of Individuals With Down Syndrome: Sudan Versus Italy", Cleft Palate Craniofac J, Vol. 52, No. 5, 2015, pp. 588-96, http://dx.doi.org/10.1597/14-082.

[22] C. Sforza et al. "Morphometry of the orbital region soft tissues in Down syndrome", J Craniofac Surg, Vol. 23, No.1, 2012, pp. 198-202, http://dx.doi.org/10.1097/SCS.0b013e3182418eba.

[23] C. Dolci et al. "3D Craniofacial Morphometric Analysis of Young Subjects with Marfan Syndrome: A Preliminary Report", Proc 6th Int Conf 3D Body Scanning Technol Lugano, Switzerland, 27-28 Oct 2015, pp. 54-60, http://dx.doi.org/10.15221/15.054.

[24] C. Dolci et al. "3D Morphometric Evaluation of Craniofacial Features in Adult Subjects with Marfan Syndrome", Proc 7th Int Conf 3D Body Scanning Technol Lugano, Switzerland, 30 Nov-1 Dec 2016; pp. 98104, http://dx.doi.org/10.15221/16.098.

[25] V. Pucciarelli et al. "Three-Dimensional Craniofacial Features of Glut1 Deficiency Syndrome Patients", Proc 6th Int Conf 3D Body Scanning Technol Lugano, Switzerland, 27-28 Oct 2015, pp. 61-7, http://dx.doi.org/10.15221/15.061.

[26] V. Pucciarelli et al. "The Face of Glut1-DS Patients : A 3D Craniofacial Morphometric Analysis", Clin Anat, Vol. 30, 2017, pp. 644-52, http://dx.doi.org/10.1002/ca.22890.

[27] C. Sforza et al. "Soft- and hard-tissue facial anthropometry in three dimensions: what's new. J Anthropol Sci, Vol.91, 2013, pp. 159-84, http://dx.doi.org/10.4436/jass.91007.

[28] V. Pucciarelli et al. "Facial Evaluation in Holoprosencephaly", J Craniofac Surg, Vol. 28, No.1, 2017, pp. e228, http://dx.doi.org/10.1097/SCS.0000000000003171.

[29] http://www.canfieldsci.com/imaging-systems/vectra-m3-3d-imaging-system/accessed on July 20, 201.

[30] FE. Jansen et al. "Severe myoclonic epilepsy of infancy (Dravet syndrome): recognition and diagnosis in adults", Neurol, Vol. 67, No. 12, 2006, pp. 2224-6, http://dx.doi.org/10.1212/01.wnl.0000249312.73155.7d.

[31] EC. Wirrell "Treatment of Dravet Syndrome", Can J Neurol Sci, Vol. 43, Suppl. 3, 2016, pp. S13-8, http://dx.doi.org/10.1017/cjn.2016.249.

[32] M de Menezes et al. "Accuracy and reproducibility of a 3-dimensional stereophotogrammetric imaging system", J Oral Maxillofac Surg, Vol. 68, No. 9, 2010, pp. 2129-35, http://dx.doi.org/10.1016/j.joms.2009.09.036.

[33] KJ Nolan et al. "Does Dravet syndrome have a recognizable face? ", Pediatr Neurol, Vol. 45, No. 6, 2011, pp. 392-4, http://dx.doi.org/10.1016/j.pediatrneurol.2011.09.008.

[34] R Corcuera-Flores et al. "A systematic review of the oral and craniofacial manifestations of cri du chat syndrome", Clin Anat, Vol. 29, No. 5, 2015, pp. 555-60, http://dx.doi.org/10.1002/ca.22654. 